\title{
Animat, un animal artificial
}

\author{
Animat, an artificial animal
}

\author{
ANTONIO BENÍTEZ \\ Universidad Complutense de Madrid (España)
}

Recibido: 7-12-2012

Aprobado definitivamente: 16-2-2013

\section{RESUMEN}

En este trabajo presento algunos experimentos realizados con el modelo Animat. En este modelo es necesario distinguir el entorno o mundo, por un lado, y el autómata (Animat), por otro. En todos estos experimentos, el entorno es el mismo. Lo que cambia de uno a otro es la estructura y la dinámica del sistema que llamamos «Animat». En la primera sección expondré la composición del entorno y, en las siguientes, la composición de Animat en cada caso.

PALABRAS CLAVE

VIDA ARTIFICIAL, INTELIGENCIA ARTIFICIAL BIOINSPIRADA, ANIMAT MODE$\mathrm{LO} 2$

\section{ABSTRACT}

In this paper I present some experiments realized with the model Animat. In this model it is necessary to distinguish between the environment or world and the automaton (Animat). In all these experiments, the environment is the same. What changes, from one to other one, is the structure and dynamics of the system that we call «Animat». In the first section I will expose the composition of the environment. In the following ones, I will present Animat's composition in every case.

KEYWORDS

ARTIFICIAL LIFE, BIO-INSPIRED ARTIFICIAL INTELLIGENCE, THE ANIMAT MODEL 2 


\section{ENTORNOS}

El ENTORNO ESTÁ FORMADO POR UNA MATRIZ DE DATOS de $f$ filas y $c$ columnas. En todos los experimentos que estudiaremos, las filas serán 50 y las columnas también 50. En cada posición de la matriz $\left(\mathrm{f}_{\mathrm{i}}, \mathrm{c}_{\mathrm{j}}\right)$ hay un objeto.

Cada objeto, excepto Animat, es una instancia o ejemplar de la clase elemento\%, una subclase de la clase color\%. Por esto último, cada ejemplar tiene un valor de rojo, verde, azul y de opacidad, es decir, valores R G B O. Además, cada ejemplar tiene otras propiedades: estado, dureza y olor. La propiedad estado se utiliza sólo en un entorno cambiante por computación local (autómatas celulares). Dureza es una propiedad de cada objeto correlativa al receptor sensitivo tacto. Por último, olor es una propiedad cuyo valor es un objeto de la clase olor\%. A continuación sintetizo estas propiedades y sus posibles valores:

Tabla: Distintos tipos de objetos

\begin{tabular}{|c|c|c|c|c|}
\hline nombre & estado & dureza & olor & color \\
\hline blanco & 0 & 0 & olor0 & 2552552551.0 \\
\hline rojo & 1 & 5 & olor0 & 255001.0 \\
\hline verde & 2 & 2 & olor2 & 025501.0 \\
\hline azul & 3 & 7 & olor0 & 002551.0 \\
\hline negro & 4 & 10 & olor0 & 0001.0 \\
\hline
\end{tabular}

De los anteriores tipos de objetos, hay dos (blancos y verdes) que son especiales, y son especiales por las siguientes razones: $1^{\mathrm{a}}$, porque sólo con el tipo de objetos blanco es posible intercambiar la posición. Es decir, Animat sólo puede avanzar a una posición donde haya un blanco; $2^{\mathrm{a}}$, porque Animat sólo puede comer objetos del tipo verde.

La matriz de datos del Entorno contiene toda la información, incluida la propia de Animat porque es un objeto más del Entorno. Por ello, en cada iteración el Entorno tiene una cierta configuración.

Todos los objetos excepto Animat están quietos porque carecen de dinamismo propio (Entorno-0). Animat sí tiene dinamismo propio. Sin embargo, veremos experimentos en que el dinamismo del entorno es distinto (Entorno-celular).

Tanto el intercambio de posiciones de Animat y un blanco como el cambio que implica la desaparición de un verde (sustituido por un blanco) porque es comido no se produce hasta que esta información no se asienta en la matriz de datos. 


\section{ANIMAT DEL PRIMER EXPERIMENTO}

En este experimento Animat está dotado de un receptor sensitivo con el que contacta con el entorno, recibiendo de éste tres estímulos. Estos estímulos son procesados por una red formada por 15 fotorreceptores, cada uno de los cuales producirá una respuesta cuyos valores posibles son 0 o 1 . La salida de esta retina es procesada por una red de tres capas del tipo perceptrón multicapa. La salida de esta red es una orden motora, que constituye la entrada de los efectores de Animat. Los efectores están constituidos por dos tipos de capacidades: una, de cambio de posición; otra, de giro sobre su propio eje con el consiguiente cambio de orientación.

\section{II.1. RECEPTOR SENSITIVO}

La retina está formada por fotorreceptores. Cada fotorreceptor es un ejemplar de una clase de nombre fotorreceptor\%. Lo característico de esta clase consiste en que cada ejemplar pertenece a un tipo por el que es sensible al rojo, verde, azul, blanco o negro. Los fotorreceptores se agrupan en tres unidades de 5 ejemplares. Con cada una de estas agrupaciones se analiza un estímulo. La salida de cada agrupación sigue, por tanto, el esquema siguiente:

\section{Rojo Verde Azul Blanco Negro}

\section{II.1.1. COMPUTACIÓN DE UN FOTORRECEPTOR}

La computación de un fotorreceptor depende del tipo al que el ejemplar pertenece y sigue la regla siguiente:

1. si la composición RGB del estímulo es igual al tipo RGB del fotorreceptor, devuelve 1

2. en otro caso, devuelve 0 .

\section{II.1.2. ESTRUCTURA DE CADA AGRUPACIÓN DE FOTORRECEPTORES}

Cinco ejemplares de fotorreceptor\% conforman una agrupación de fotorreceptores según el esquema siguiente:

$$
\text { tipo_rojo tipo_verde tipo_azul tipo_blanco tipo_negro }
$$

La computación de una agrupación consiste en lo siguiente:

- Cada fotorreceptor pertenece a un tipo; cada estímulo es un objeto perteneciente a un tipo (rojo, verde, azul, blanco o negro). 
- Si coinciden el tipo del fotorreceptor y el del objeto, la neurona fotorreceptora devuelve 1; en caso contrario, 0 .

- El esquema de la salida de una agrupación de fotorreceptores es:

$(0 / 10 / 10 / 10 / 10 / 1)$

\section{II.1.3. LA RED DE FOTORRECEPTORES}

La retina está formada por tres agrupaciones de fotorreceptores según el esquema siguiente:

$$
\text { agrupación_1 agrupación_2 agrupación_3 }
$$

La computación de la red puede ser expresada así:

- Cada uno de los tres estímulos (un objeto de un cierto tipo) estimula una de las tres agrupaciones de fotorreceptores.

- La salida de la retina está formada, por tanto, por las salidas de las agrupaciones.

- La salida de la retina puede tratarse, según convenga, como una única lista de 15 elementos o como una lista formada por tres listas de 5 elementos cada una.

\section{II.2. CONTROL CENTRAL}

El cerebro está formado por una única red de neuronas artificiales. Esta red, del tipo perceptrón multicapa, está formada por tres capas:

1. Capa de entrada formada por 15 neuronas de entrada. Cada una de estas neuronas recibe uno de los 15 valores de la salida de la retina.

2. Capa oculta formada por 15 neuronas. Cada una de estas está conectada con cada una de las 15 neuronas de la capa de entrada.

3. Capa de salida formada por 4 neuronas, cada una conectada con cada una de las 15 neuronas de la capa oculta.

Los parámetros de la red, pesos y umbrales, han sido obtenidos mediante la aplicación de un Algoritmo Genético. La red produce una orden motora según el esquema siguiente:

Tabla 2: Esquema orden motora

\begin{tabular}{|l|l|l|l|}
\hline vec_1 & vec_2 & vec_3 & giro \\
\hline blanco & 0 & 0 & 0 \\
\hline 0 & blanco & 0 & 0 \\
\hline 0 & 0 & blanco & 0 \\
\hline
\end{tabular}




\begin{tabular}{|l|l|l|l|}
\hline 0 & 0 & 0 & 1 \\
\hline
\end{tabular}

Si hay un blanco en cualquiera de las tres posiciones de los estímulos, el esquema seguido será uno de las tres primeras filas. En ese caso, no habrá giro (valor 0 ). En cualquier otro caso, cuando no hay un blanco entre los estímulos, el esquema seguido será el de la última fila, y habrá giro porque su valor será 1 .

\section{II.3. EFECTORES}

Animat tiene tanto la capacidad de avanzar a una posición vacía (donde hay un blanco) como la capacidad de girar cambiando de orientación. Mientras que la capacidad de avanzar es una locomoción o cambio de lugar, la capacidad de girar no es una locomoción puesto que no hay cambio de lugar, pero sí es un movimiento que produce un cambio de orientación en el aquí de Animat.

\section{ANIMAT DEL SEGUNDO EXPERIMENTO}

En este segundo experimento Animat está dotado de tres receptores sensitivos: vista, olfato y tacto. Y tiene un efector más: la acción de comer. No es la vista sino el tacto y el olfato los receptores que proporcionan información, de modo distinto, sobre comida.

Otra novedad es que cada receptor sensitivo se prolonga en una red de tipo ART cuya función es clasificar la información analizada por cada receptor. Si se conciben estas redes como un córtex-visual, córtex-táctil y córtex-olfativo, los órganos sensoriales se conectan con zonas corticales. La salida de cada uno de esas tres zonas corticales es el input de una red de tipo perceptrón multicapa.

Además he añadido otra zona cortical. Esta zona esta formada por un sensor de energía, sensor que mide la energía existente en el sistema y un autómata de órdenes, que discrimina entre necesidades y construye la orden motora. El sensor está, en cada iteración o instante temporal, en un estado: 0, 1, y 2. Según el estado de este sensor, la orden motora se construye en esta zona cortical según la idea siguiente: predomina la vista, si no hay necesidad de comer; predomina el tacto, si hay poca necesidad de comer; y predomina una combinación de tacto y olfato, si hay mucha necesidad de comer.

\section{III.1. RECEPTORES SENSITIVOS}

Animat dispone de tres receptores sensitivos: retina, tacto y olfato. En el diseño del tacto y el olfato he seguido el modelo de la retina del primer experimento. Por tanto, hay neuronas fotorreceptoras, receptoras de lo tocado y quimiorreceptoras, estas dos últimas siguen la misma idea ensayada en los fotorreceptores. Comparo sintéticamente la estructura y composición de la retina, tacto y olfato en los apartados siguientes. 


\section{III.1.1. ESTÍMULOS Y RECEPTORES SENSITIVOS}

La vista es estimulada por los tres objetos que están frente a Animat. Esos mismos objetos son los que Animat toca. Sin embargo, Animat es capaz de oler cada uno de los ocho vecinos que le rodean (vecindad de Moore).

La retina está formada por tres agrupaciones de fotorreceptores. Una agrupación analiza un estímulo.

Tabla 3: Salida de una Agrupación de fotorreceptores

\begin{tabular}{|l|l|l|l|l|}
\hline rojo & verde & azul & blanco & negro \\
\hline $1 / 0$ & $1 / 0$ & $1 / 0$ & $1 / 0$ & $1 / 0$ \\
\hline
\end{tabular}

El tacto está formado por tres agrupaciones de sensores del tacto. Cada agrupación analiza un estímulo.

Tabla 4: Salida de una Agrupación de sensores táctiles

\begin{tabular}{|l|l|l|l|l|}
\hline dureza-rojo & dureza-verde & dureza-azul & dureza-blanco & dureza-negro \\
\hline $1 / 0$ & $1 / 0$ & $1 / 0$ & $1 / 0$ & $1 / 0$ \\
\hline
\end{tabular}

El olfato está formado por ocho agrupaciones de quimiorreceptores. Cada agrupación analiza igualmente un estímulo.

Tabla 5: Salida de una Agrupación de quimiorreceptores

\begin{tabular}{|l|l|l|l|l|}
\hline olor-rojo & olor-verde & olor-azul & olor-blanco & olor-negro \\
\hline $1 / 0$ & $1 / 0$ & $1 / 0$ & $1 / 0$ & $1 / 0$ \\
\hline
\end{tabular}

III.2. REGIÓN CORTICAL DE LOS SENTIDOS

Cada uno de los tres receptores sensitivos se conecta con una red ART. Como es sabido, este tipo de redes es eminentemente clasificatoria. La salida de una red ART es el identificador de la categoría en que la red ha clasificado el input recibido.

\section{III.3. REDES DE DISCRIMINACIÓN. PRE-ÓRDENES MOTORAS}

Cada uno de los tres córtex sensitivos conecta con una red del tipo perceptrón multicapa. La función de estas redes es discriminar entre dos categorías de estímulos: los que representan una facilidad y los que representan una dificultad respecto a una necesidad prioritaria. 
Así, cuando Animat no necesita comer, sí necesita moverse: es un semoviente. En estos casos Animat se guía exclusivamente por su vista. Y su vista le informa de dos tipos posibles de estímulos: lugares vacíos (blancos) ofacilidades para el avance; cosas (objetos no-blancos) o dificultades para el avance.

Cuando Animat necesita comer, aunque su energía sea aún positiva, se guía por el tacto para buscar comida y por la vista para cambiar de lugar. El tacto, como la vista, le informa de dos categorías de estímulos: comida (verdes) o facilidad para incrementar la energía del sistema; resto de objetos (no comida) o dificultades para incrementar la energía del sistema.

En las situaciones en que Animat necesita comer urgentemente, se guía por el tacto y el olfato para encontrar comida, y por la vista para cambiar de lugar. Si el tacto detecta comida, Animat come. Si el olfato detecta comida y el tacto no, Animat gira (ver tabla 8). Si ninguno de los dos sentidos, tacto y olfato, detecta comida, Animat se mueve según lo que informa su vista.

\section{III.3.1. RED DE DISCRIMINACIÓN VISUAL}

- Perceptrón de 3 capas

- Capa de entrada: 3 neuronas; Capa oculta: 3 neuronas, función de escalón; Capa de salida: 3 neuronas, función de escalón

- El ajuste de parámetros se ha hecho mediante un Algoritmo Genético

- Salidas posibles:

Tabla 6: Red de discriminación visual

\begin{tabular}{|l|l|l|l|}
\hline 1 & 0 & 0 & avanzar $\rightarrow$ vecino \\
\hline 0 & 1 & 0 & avanzar $\rightarrow$ vecino \\
\hline 0 & 0 & 1 & avanzar $\rightarrow$ vecino \\
\hline 0 & 0 & 0 & girar \\
\hline
\end{tabular}

III.3.2. RED DE DISCRIMINACIÓN TÁCTIL

- Perceptrón de 3 capas

- Capa de entrada: 3 neuronas; Capa oculta: 3 neuronas, función de escalón; Capa de salida: 3 neuronas, función de escalón

- El ajuste de parámetros se ha hecho mediante un Algoritmo Genético

- Salidas posibles:

Tabla 7: Red de discriminación táctil

\begin{tabular}{|l|l|l|l|}
\hline 1 & 0 & 0 & comer $\rightarrow$ vecino \\
\hline 0 & 1 & 0 & comer $\rightarrow$ vecino \\
\hline 0 & 0 & 1 & comer $\rightarrow$ vecino \\
\hline 0 & 0 & 0 & girar \\
\hline
\end{tabular}




\section{III.3.3. RED DE ANÁLISIS OLFATIVO}

- Perceptrón de 3 capas

- Capa de entrada: 8 neuronas; Capa oculta: 8 neuronas, función de escalón; Capa de salida: 8 neuronas, función de escalón

- El ajuste de parámetros se ha hecho mediante un Algoritmo Genético

- Algunas salidas posibles:

Tabla 8: Red de análisis olfativo

\begin{tabular}{|l|l|l|l|l|l|l|l|l|l|}
\hline & & & & & & & & acción \\
\hline 1 & 0 & 0 & 0 & 0 & 0 & 0 & 0 & acción-según tacto \\
\hline 0 & 1 & 0 & 0 & 0 & 0 & 0 & 0 & acción-según tacto \\
\hline 0 & 0 & 1 & 0 & 0 & 0 & 0 & 0 & acción-según tacto \\
\hline 0 & 0 & 0 & 1 & 0 & 0 & 0 & 0 & girar a derechas \\
\hline 0 & 0 & 0 & 0 & 1 & 0 & 0 & 0 & girar a derechas \\
\hline 0 & 0 & 0 & 0 & 0 & 1 & 0 & 0 & girar a derechas \\
\hline 0 & 0 & 0 & 0 & 0 & 0 & 1 & 0 & girar a izquierdas \\
\hline 0 & 0 & 0 & 0 & 0 & 0 & 0 & 1 & girar a izquierdas \\
\hline
\end{tabular}

\section{III.4. NECESIDADES Y ÓRDENES MOTORAS}

Animat está concebido como un animal artificial dotado inicialmente de una cierta cantidad de energía. Con cada acción realizada, Animat pierde un poco de esa energía.

Mientras que la cantidad de energía es superior a 100, el sensor de energía (estado = 0) no detecta necesidad de incrementar la energía del sistema. Cuando ese es el caso, Animat no tiene necesidad de comer. En estas situaciones, Animat se guía exclusivamente por su vista.

Si la energía del sistema baja de 100 (entre 100 y 0), el sensor de energía se activa y su estado es 1. Mientras que este sea el caso, Animat tiene como prioridad comer un verde. En estas situaciones, Animat se deja guiar por el tacto para encontrar comida y por la vista para avanzar o girar.

Una tercera situación es aquella en que la energía del sistema es negativa (pero mayor que -200). El estado del sensor de energía es 2. En estas situaciones, Animat debe comer urgentemente porque si la energía del sistema baja de -200, Animat muere. Cuando el estado del sensor de energía es 2, Animat se guía por el tacto y el olfato para encontrar comida y por la vista para cambiar de lugar. En aquellas situaciones en que el tacto no detecta comida y el olfato 
huele comida en algunos de los vecinos que no están frente a Animat, gira a izquierda o a derecha según la ubicación del estímulo olfativo.

Dependiendo, pues, del estado del sensor de energía, Animat tendrá una necesidad prioritaria u otra. La orden motora dependerá, en cada caso, tanto del entorno como del estado del sensor interno de energía.

He organizado estas ideas en un módulo cuya computación sigue los criterios siguientes:

- Estado del sensor de energía $=0$, ORDEN $=$ salida de la red de discrimi nación visual

- Estado del sensor de energía $=1$, ORDEN $=$ salida de la red de discriminación táctil o salida de la red de discriminación visual

- Estado del sensor de energía $=2$, ORDEN $=$ salida de la red de discriminación táctil o salida de la red de análisis olfativo o salida de la red de discriminación visual

\section{III.4.1. MEMORIA DE LA ORDEN PREVIA}

Cuando Animat lleva a cabo una acción de comer, la orden motora correspondiente se almacena en la memoria reciente (propiedad orden_ensayada). Si la acción no es de comer, no se almacena la orden motora correspondiente (orden_ensayada $=$ falso).

Si la orden motora es de avanzar y orden_ensayada $\neq$ falso, se sustituye dicha orden motora por la contenida en orden_ensayada. En estos casos Animat avanza al lugar ocupado por el último verde comido.

Se trata, pues, de un mecanismo de inhibición posible y sustitución de una orden motora.

\section{III.5. EFECTORES}

Animat puede llevar a cabo tres acciones básicas: cambiar de lugar, comer y girar. El cambio de lugar produce un cambio del aquí de Animat y, con ello, de la perspectiva que Animat tiene del Entorno. Al girar no hay cambio del aquí pero sí de la perspectiva que tiene del Entorno. Comer no produce un cambio en el aquí de Animat, pero sí en su perspectiva del Entorno.

El cuadro de acciones posibles es el siguiente:

Tabla: Acciones posibles

\begin{tabular}{|l|l|}
\hline orden motora & acción \\
\hline$\left(\begin{array}{llll}0 & 1 & 0 & 0\end{array}\right)$ & avanzar $\rightarrow$ vecino \\
\hline$\left(\begin{array}{llll}0 & 0 & 1 & 0\end{array}\right)$ & avanzar $\rightarrow$ vecino \\
\hline
\end{tabular}




\begin{tabular}{|l|l|l|}
\hline$\left(\begin{array}{llll}0 & 0 & 0 & 1\end{array}\right)$ & avanzar $\rightarrow$ vecino \\
\hline$\left(\begin{array}{lllll}1 & 1 & 0 & 0\end{array}\right)$ & comer $\rightarrow$ vecino \\
\hline$\left(\begin{array}{lllll}1 & 0 & 1 & 0\end{array}\right)$ & comer $\rightarrow$ vecino \\
\hline$\left(\begin{array}{lllll}1 & 0 & 0 & 1\end{array}\right)$ & comer $\rightarrow$ vecino \\
\hline$\left(\begin{array}{lllll}0 & 0 & 0 & 0\end{array}\right)$ & girar a derechas \\
\hline$\left(\begin{array}{lllll}1 & 0 & 0 & 0\end{array}\right)$ & girar a izquierdas \\
\hline
\end{tabular}

III.6. GRAFO DE CONEXIONES

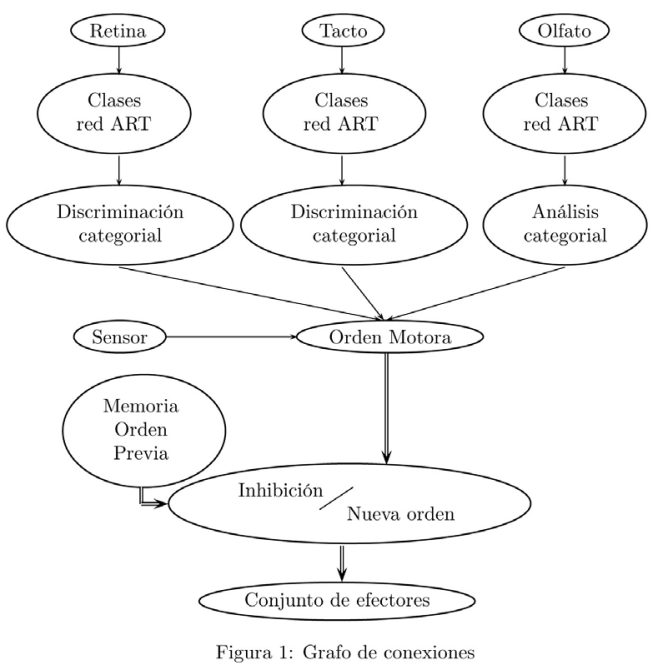

IV. DisCUSIÓN DE IDEAS

\section{IV.1. EL ENTORNO COMO TOTALIDAD}

Cuando se contempla la ventana del monitor, se está viendo el modelo experimental entero, es decir, se está viendo el Entorno como una totalidad entre cuyas partes Animat es una más. Esta totalidad puede ser entendida, en primer lugar, como una colección de objetos.

Pero esa totalidad, en segundo lugar, tiene una forma determinada. Hemos visto en la primera sección que al Entorno corresponde una matriz bidimensional de datos. Por ello, cada posición queda determinada por un par (fila, columna) que identifica un posible lugar en el espacio del Entorno. El Entorno es así una estructura ordenada de posiciones en la que han de ser dispuestos los objetos. La primera idea de una totalidad como mera colección de objetos ha de ser precisada: el Entorno es una estructura de posiciones en cada de las cuales se ubica un objeto. 
Corolario de esto es que dos objetos son distintos, son partes distintas de la totalidad, porque ocupan lugares distintos. Es decir, si suponemos dos objetos que tengan exactamente todos sus propiedades iguales, tendríamos que afirmar que no son el mismo porque han de ocupar dos posiciones distintas. Más aún, si contemplamos un objeto cualquiera en su interior, podríamos afirmar que el estar en una posición no es una propiedad que le pertenezca per se, pero en tanto que objeto del Entorno, en cuanto parte de este, cada objeto tiene necesariamente una determinación más - puramente relacional-: ocupar un lugar en el espacio del Entorno.

\section{IV.2. EL DINAMISMO DEL ENTORNO}

El Entorno, si bien es uno y una totalidad, puede cambiar de configuración, esto es, que el contenido de la matriz de datos puede cambiar de una iteración a otra. Los cambios que se producen en la configuración del Entorno están reglados. Es decir, que existe al menos una regla según la cual se producen los cambios de configuración. Dado que esa regla, o reglas, regula por igual cada cambio, se mantiene constante y uniforme en la secuencia de cambios de configuración. En este sentido, cada regla es ley.

La regla que rige el dinamismo del entorno puede ser concebida como una única ley: la del intercambio de posiciones. El intercambio puede darse entre dos objetos, un blanco y otro que no puede ser un blanco. En efecto, cuando Animat actúa cambiando de posición se produce un intercambio de posiciones.

Corolario de lo anterior es que el dinamismo ha de ser concebido como determinación (definición) de las relaciones de posición entre objetos.

Puede discutirse el corolario anterior, si bien no parece que puede hablarse de un dinamismo intrínseco a los objetos. ${ }^{1}$

\section{IV.3. ANIMAT COMO SISTEMA}

Animat es un único objeto. Sin embargo, no es un objeto simple, antes bien tiene partes. Es, pues, un objeto compuesto. Como decía del Entorno, puede decirse - en primer lugar- que Animat es una colección de partes: receptores, unidad de control y efectores.

En segundo lugar, a diferencia del Entorno, las partes de Animat no tienen una disposición espacial, excepto los receptores sensitivos: los fotorreceptores, la retina de Animat se ubica en la parte frontal, también los sensores del tacto, mientras que los quimiorreceptores se ubican en la periferia entera de Animat. Sin embargo, es claro que existe una cierta disposición entre todas las partes, puesto que en su funcionamiento Animat exige que una parte-receptores-actúe

1 No considero aquí el caso de que haya cambio por computación local, caso en que el entorno se asemeja a redes bidimensionales de autómatas celulares. 
antes de que la que la unidad de control pueda hacerlo. Lo mismo cabe decir de los efectores respecto a la unidad de control. Desde el punto de vista del funcionamiento interno de Animat, las partes tienen una disposición funcional, están ordenadas funcionalmente. Por ello, cada parte que compone Animat es una parte inseparable.

Esta disposición funcional, en tercer lugar, permite determinar cada acción de Animat como una tripla formada por los receptores, la unidad de control y los efectores -siempre en este orden-, según el esquema siguiente:

$$
\text { (estímulos, orden motora, acto efector) }
$$

De donde se sigue que cada acción de Animat responde a la idea de esquema de conducta de la Etología. Y, según esta idea, es posible determinar las correlaciones entre mecanismos desencadenadores innatos (MDI) y los actos efectores de Animat. En efecto, el acto de avanzar es relativo a la presencia de un blanco entre los estímulos, mientras que el giro se produce cuando no existe un blanco entre los estímulos. El autómata del segundo experimento permite añadir: que el comer se produce cuando no hay un blanco entre los estímulos y sí hay un verde.

En cuarto lugar, Animat produce una acción en cada iteración. Desde este punto de vista, Animat consiste en actividad incesante. Por ello, Animat no es como el resto de objetos del Entorno, sino que es un objeto cuya existencia entera consiste en producir acciones. Es, pues, un agente, si por agente se entiende un objeto que produce incesantemente acciones. La agencia de Animat queda determinada tanto por las correlaciones posibles entre los MDI y los actos efectores como por las triplas correspondientes a los esquemas de conducta posibles. También puede determinarse la agencia por las necesidades básicas o constitutivas de Animat.

Corolario de esto es que Animat es un objeto dinámico, frente al resto de objetos del Entorno que son a-dinámicos. ${ }^{2}$

Animat es, pues, un objeto compuesto; un sistema funcional de partes inseparables; y un sistema dinámico.

\section{IV.4. EL DINAMISMO DE ANIMAT}

Cuando se contempla el monitor de Animat, llama la atención la capacidad de sortear obstáculos que exhibe el autómata. Sabemos que al avanzar, Animat intercambia su posición con el primer blanco situado más a la izquierda. Esto no es determinante y puede ser considerado una propiedad irrelevante que emana local.

2 Esta afirmación no se puede mantener si el Entorno es cambiante por computación 
del programa mismo. Se puede, pues, cambiar. Supongamos que se hace de forma que Animat, si percibe más de un blanco, elija uno de ellos al azar para intercambiar posiciones. Por ello, no perdería esa capacidad de sortear obstáculos. Pero si eliminamos de Animat la capacidad de girar, entonces contemplaríamos que Animat es incapaz de seguir avanzando. Sin la posibilidad de realizar un acto de girar, además del acto de avanzar, Animat se convierte en un autómata limitado en sus posibilidades semovientes.

Por tanto, la capacidad de producir actos de giro ha de sumarse a la capacidad de avanzar para que Animat pueda exhibir el comportamiento que contemplamos.

Desde otro punto de vista, Animat puede satisfacer su objetivo último, a saber: cambiar de lugar incesantemente, gracias a la incorporación de la capacidad de girar entre sus efectores. Esta capacidad de girar, este mecanismo efector, tiene consecuencias. La primera es que Animat puede cambiar de orientación. Y con el cambio de orientación adquiere la posibilidad de tener perspectivas distintas sobre el Entorno sin necesidad de avanzar. Permaneciendo en el mismo lugar, Animat introduce una nueva perspectiva al girar. El cambio de perspectiva, producido por el acto de girar, tiene consecuencias sobre el resto del cuerpo de Animat: los estímulos visuales son otros. Por tanto, cambia su ahí: lo que hay ahí ya no es lo que había.

Según la ley de la perspectiva, ${ }^{3}$ el viviente siempre está en un aquí porque su cuerpo siempre ocupa un lugar determinado. Y dependiendo de ese aquí, sus estímulos se sitúan en un ahí. En estos experimentos Animat cumple esta ley. Por encontrarse siempre en un lugar, Animat está siempre en un aquí y los estímulos están ahí, en lo visto o tocado.

Si el avanzar es un efector necesario para que Animat sea un semoviente, al sumar la capacidad de girar Animat completa su dotación innata, puesto que con ello consigue que sus acciones logren su objetivo final: cambiar de lugar. En este sentido, Animat se adapta al Entorno o es un autómata adaptado al Entorno.

El intercambio de lugar consiste en cambiar la posición de Animat a la posición ocupada por un blanco. Pero este intercambio se produce de modo peculiar. Si nos instalamos en el interior de Animat es más fácil de entender. Si fuéramos Animat, el Entorno se nos presentaría dividido en dos clases de objetos: los que dificultan nuestro avance, los obstáculos al avance, y los que lo hacen posible o lo facilitan, los blancos o huecos vacíos. Estas dos clases son correlativas a las estructuras que componen Animat, a su composición como sistema dinámico. En este sentido, el Entorno es un Medio para Animat, en el sentido que se suele dar a la palabra medio en la Biología.

3 Vid. Antonio Benítez: Fundamentos de Inteligencia Artificial. Libro segundo: Inteligencia Artificial clásica. Madrid, Escolar y Mayo, 2011, sección 13.2.1 
Por último, si es cierto que el Entorno se compone de dos clases de objetos para Animat, entonces los estímulos son señales,${ }^{4}$ información, para la conducta de Animat. En cuanto sistema que obtiene su conducta procesando información proveniente de su Medio, creo que cabe concebir Animat como un embodied Agent.

\section{BiBLIOGRAFÍA}

BEER, R.D., Intelligence as adaptive behavior: an experiment in computational neuroethology. Academic Press, Boston, 1990.

BENÍTEZ, A. Fundamentos de Inteligencia Artificial. Libro segundo: Inteligencia Artificial clásica. Escolar y Mayo, Madrid, 2011.

BROOKS, R. A., «Elephants Don’t Play Chess», Robotics and Autonomous Systems 6, pp. 3-15, 1990. 1991. «Intelligence without representation», Artificial Intelligence 47, pp. 139-159, , Intelligence Without Reason. MIT, Memo Num. 1293, 1991.

EIBL-EIBESFELDT, I., «Adaptaciones filogenéticas en el comportamiento del hombre». En: H-G. Gadamer, P. Vogler (dir.): Nueva Antropología. Tomo 2: Antropología biológica. Trad. de Margarida Costa. Ediciones Omega, Barcelona, pp. 3-55, 1976.

, El hombre preprogramado. Trad. esp. Pedro Gálvez. Alianza editorial, Madrid, 1977.

, Biología del comportamiento humano. Manual de etología humana. Trad. esp. Francisco Giner y Luis Cencillo. Alianza editorial, Madrid, 1993.

WILSON, S. W., «Knowledge Growth in an Artificial Animal». En: J.J. Grefenstette (Ed.), Proceedings of an International Conference on Genetic Algorithms and Their Applications, Lawrence Erlbaum Assoc., Hillsdale, NJ, pp. 16-23, 1985. , «The Animat Path to AI». En: J. A. Meyer, S. W. Wilson (Eds.), Animals to Animats: Proceedings of The First International Conference on Simulation of Adaptive Behavior. The MIT Press/Bradford Books, Cambridge, MA, pp. $15-21,1991$.

4 Vid. Antonio Benítez: Fundamentos de Inteligencia Artificial. Libro segundo: Inteligencia Artificial clásica. Madrid, Escolar y Mayo, 2011, pp. 238-239. 
Antonio Benítez López es Profesor Titular en el Departamento de Lógica y Filosofía de la Ciencia. Facultad de Filosofía. Universidad Compluyense de Madrid.

Líneas de investigación:

Lógica, Inteligencia Artificial

Publicaciones recientes:

(2009) Apuntes de Historia y Filosofía de la Lógica. Madrid: Cersa. 320 páginas.

(2011) Fundamentos de Inteligencia Artificial. Libro segundo: Inteligencia Artificial clásica. Escolar y Mayo, Madrid.

Correo electrónico: abenitez@filos.ucm.es 